\title{
Reflexión y nuevos retos de Unibasq, Agencia de Calidad del Sistema Universitario Vasco
}

\author{
Reflection and new challenges in Unibasq, the \\ Basque University System Quality Assurance
} Agency

Eva Fernández de Labastida Amurrio Responsable de evaluación Unibasq Email: eva.fdzlabastida@unibasq.org

\section{Eva Ferreira García}

Directora de la Agencia Unibasq Email: eva.ferreira@unibasq.org

Aitor Zuramendi Isla

Adjunto a la Dirección Unibasq

Email: aitor.zurimendi@unibasq.org

\section{Resumen}

Unibasq es la Agencia de Calidad del Sistema Universitario Vasco. Creada en 2004, su trayectoria, al igual que en otras agencias, es paralela a la construcción del Espacio Europeo de Educación Superior (EEES). En este artículo analizamos la evolución de Unibasq, los cambios de su marco legal y sus competencias, y la visión actual y de futuro. La evolución refleja el aumento continuo de la actividad y la consolidación de la Agencia como asesora en el Sistema Universitario Vasco. Los cambios en el marco legal refrendan los valores de la agencia de independencia y objetividad y han posibilitado su reconocimiento europeo. Finalmente, presentamos las grandes líneas del Plan Estratégico 2016-19, fundamentadas en la simplificación de procedimientos, proyección social de la actividad universitaria, asesoramiento y apoyo a la internacionalización de 
las universidades vascas y, naturalmente, en la mejora de la propia agencia. La buena acogida del plan estratégico por los grupos de interés avala y afianza la vocación de la agencia por ser reconocida por las instituciones de educación superior, la comunidad universitaria y la sociedad como un agente que ayuda a mejorar la calidad del sistema universitario vasco, así como a su internacionalización y proyección social.

\section{Palabras clave}

Quality Assurance Agency, EHEA, University, Higher Education, Evaluation, centros comunitarios de iformación jurídaca, dificultades.

\section{Abstract}

Unibasq is the Basque University System Quality Assurance Agency. Created in 2004, its evolution, as for other agencies, is parallel to the European Higher Education Area (EHEA). In this article, we analyze the Unibasq evolution, the legal framework changes, the presente situation and the future vision. The evolution reflects the continuous increase of its activity and its consolidation as advisor in the Basque University System. The changes in the legal framework countersign the agency's values, independence and objectivity and have enabled its European recognition. Finally, we present the main lines of the Strategic Plan 2016-19, based on procedures simplification, social projection of the university activity, assessment and support for the Basque universities internationalization and, naturally, also on the agency improvement. The good reception of the strategic plan by the stakeholders endorses and secures the agency vocation of being recognized by the higher education institutions, the university community and the society as a supporter of the quality, internationalization and social projection in the Basque University System.

\section{Key words}

Clinical legal education, community legal centre, difficulties. 


\section{Introducción: EL EEES Y SU IMPLANTACIÓN EN ESPAÑA}

La construcción del Espacio Europeo de Educación Superior (EEES) supuso un hito importante para el desarrollo de la educación superior en nuestro país. En este proyecto aparece como elemento central la garantía de la calidad de la actividad universitaria, para lo que se crearon las agencias de evaluación y acreditación de la calidad como sujetos activos de esta labor.

En la Ley Orgánica de Universidades (2001) se da carta de naturaleza a las agencias de evaluación de la actividad universitaria, como organismos responsables de la evaluación de la calidad de las universidades. Además de la agencia estatal, las Comunidades Autónomas con competencia en materia de educación superior, como es el caso de Euskadi, constituyeron la suya propia.

Unibasq es un ente público de derecho privado adscrito al departamento del Gobierno Vasco competente en materia de universidades que tiene como objetivo la evaluación, acreditación y la certificación de la calidad en el ámbito del Sistema Universita rio Vasco (en adelante, SUV), considerando el marco español e internacional. Se constituyó en 2006, y posteriormente se adaptó para cumplir los estándares europeos en la Ley $13 / 2012$, donde se le dio la actual denominación. Su propósito es la evaluación, acreditación y certificación de la calidad del SUV, de acuerdo con los estándares españoles e internacionales. También puede realizar evaluaciones, acreditaciones y certificaciones de actividades fuera de la Comunidad Autónoma Vasca, mediante acuerdos previamente suscritos con entidades extranjeras. Además, la agencia puede establecer relaciones de cooperación, colaboración, reconocimiento de los procesos de evaluación, acreditación y certificación e intercambio de información con otras agencias autonómicas, estatales o extranjeras que tengan atribuidas funciones de evaluación, acreditación y certificación de la calidad en la educación superior.

La finalidad principal de la agencia es la mejora de la calidad del sistema universitario vasco para que esté bien posicionado en el EEES. Para ello, además de las actividades evaluadoras y certificadoras, asesora a los agentes que participan en el Sistema Universitario Vasco, y proporciona información a la sociedad sobre el mismo.

Desde septiembre de 2014, Unibasq es miembro de pleno derecho de ENQA (the European Association for Quality Assurance in Higher Education), y desde finales de 2014 se encuentra formalmente incluida en EQAR (European Quality Assurance Register for Higher Education). La inscripción supone el reconocimiento para llevar a cabo su actividad de evaluación de la calidad universitaria de acuerdo con criterios internacionalmente reconocidos e implica que, en adelante, las nuevas titulaciones que se impartan en las universidades vascas no tendrán que ser evaluadas por instancias externas al propio País Vasco.

La nueva situación de Unibasq junto con el hecho de que ya han pasado diez años desde la puesta en marcha del proceso de Bolonia, han marcado un proceso de reflexión en la segunda mitad del 2015, conducentes a la elaboración de un nuevo Plan Estratégico. 
La elaboración de este plan no ha sido ajena a la reflexión generalizada que se está realizando en torno a la puesta en marcha del proceso de Bolonia y sus consecuencias. Entre otras, este proceso ha aumentado significativamente la rendición de cuentas de las universidades y la importancia de los resultados del aprendizaje y, por tanto, de las unidades de medida. Algunos países, como Reino Unido, tienen una trayectoria más larga en los procesos de evaluación y rendición de cuentas. Lomas (1999), Jackson (2000) y Cartwright (2007) son ejemplos de trabajos que analizan dicha trayectoria.

En lo que al sistema universitario español se refiere, en la última década han aparecido análisis sobre el proceso de Bolonia. En este sentido Rue (2009) aborda una reflexión acerca de la naturaleza del proceso de cambio de la Educación Superior (ES) en España, en su vertebración en el Espacio Europeo de Educación Superior, partiendo de la idea de que este proceso implica un cambio de naturaleza cultural, con importantes implicaciones en el ámbito de lo político, de lo organizativo y en el de la responsabilización de los agentes participantes o involucrados en él y en el del tipo de apoyo que se requiere.

Más recientemente, Casanova (2015) considera la función supervisora como eje sobre el cual pueden girar los cambios, reformas e innovaciones educativos de los diferentes sistemas, siempre que se le atribuyan las funciones y competencias adecuadas para intervenir con autoridad en los momentos y situaciones pertinentes. Considera que la inspección debe avalar la calidad educativa del sistema juntamente con la dirección de los centros, especialmente en situaciones de cambio, innovación y reforma.

Ion y Cano (2011) realizan una descripción sobre la situación del aseguramiento de la calidad en las universidades españolas, basada en qué prácticas se impulsan entre académicos y académicas, cómo se evalúan dichas prácticas, y cuáles son las fortalezas y debilidades de las prácticas basadas en competencias.

Todo esto ha conducido a un análisis sobre el proceso en general y sobre las medidas de evaluación de la docencia en particular. Así Ruiz (2005) presenta una propuesta para integrar los resultados de la evaluación de la docencia en los planes de mejora de la calidad de la Universidad de Jaen, bajo el reconocimiento de que "la actividad docente es una tarea compleja, multidimensional, en la que el profesor puede asumir diferentes roles, adoptando enfoques diferentes respecto a la enseñanza". Por su parte Molero (2004) establece que la medida de la calidad de la docencia universitaria basada en estudios de satisfaccion de los estudiantes no ofrece resultados concluyentes. Esas encuestas no pueden ser la única manera de evaluar la docencia.

Calderón y Escalera (2008) realizan una valoración del programa DOCENTIA y de la lectura del documento (DOCENTIA) preocupa que no se refiera ni afronte la situación real de la universidad española, con una falta clara de recursos y un profesorado con bajos salarios, escasos o nulos incentivos y cuya actividad profesional está escasamente valorada socialmente.

Así, Unibasq ha afrontado la nueva etapa definiendo nuevos retos de forma realista, considerando su situación de partida, la evolución realizada y las características del Sistema Universitario Vasco, receptor principal de la actividad de la agencia.

El resto del artículo se divide en tres secciones, marco legal y organizativo de Unibasq, 
evolución de la actividad realizada por la agencia en el periodo 2004-2015 y nuevos retos en el plan estratégico 2016-2019.

\section{Marco legal y organizativo de UNIBASQ}

\subsection{Evolución normativa y funciones}

La Ley Orgánica de Universidades de 21 de Diciembre de 2001 tenía como objetivo fundamental la mejora de la calidad de las universidades españolas para reforzar su competitividad y mejorar su posición en el Espacio Europeo de Educación Superior, ocupándose para ello de la calidad de sus titulaciones, de su profesorado y de su sistema de gestión. Para ello incrementó la capacidad de decisión de las universidades dotándolas de nuevas herramientas para poder competir. Así por ejemplo, se les otorgó más autonomía en la contratación de profesorado y se les facilitó instrumentos jurídicos que facilitaran la movilidad internacional tanto del alumnado como del profesorado. Con este mismo objetivo, se atribuyó a las Comunidades Autónomas la regulación del régimen jurídico y retributivo del profesorado contratado, la capacidad para establecer retribuciones adicionales para el profesorado, la aprobación de programas de financiación plurianual conducentes a contratos programa y la evaluación de la calidad de las Universidades de su ámbito de responsabilidad.

Por tanto, su objetivo es la mejora continua del sistema universitario en cada una de sus vertientes, generalizando la cultura de la evaluación tanto a nivel de las titulaciones ofertadas, como de la docencia e investigación del profesorado, como de los sistemas de gestión de las Universidades. Para poder llevar a cabo tales evaluaciones se contempla por primera vez la figura de las agencias de calidad de la educación superior, en la esperanza de que su trabajo proporcione "una información adecuada para la toma de decisiones, tanto a los estudiantes a la hora de elegir titulaciones o centros como a los profesores y a las Administraciones públicas al elaborar las políticas educativas que les corresponden" (Exposición de Motivos). En concreto, se atribuyen estas funciones a ANECA (Agencia Nacional de Evaluación de la Calidad y Acreditación), o a otras agencias que puedan constituir algunas Comunidades Autónomas con competencia para ello, siempre que cumplan los estándares internacionales.

En el caso de Euskadi, su Estatuto de Autonomía le otorga competencias en materia de educación, por lo que en 2005 procedió a crear su propia agencia al amparo de la Ley 3/2004 del Sistema Universitario Vasco, que se denominó inicialmente Uniqual - Agencia de Evaluación de la Calidad y Acreditación del Sistema Universitario Vasco. Transcurridos unos años, se estimó oportuno una reforma de la Agencia, con el objetivo de adaptar su estructura y funcionamiento a los criterios y directrices europeos recomendados para las agencias de garantía externa de calidad, que son la guía básica que debe seguir cualquier agencia para el reconocimiento de sus evaluaciones y de su actividad en el ámbito europeo. Con esta declarada finalidad se aprobó tanto la Ley 13/2012 de Unibasq-Agencia de Calidad del Sistema Universitario Vasco, que cambió además su denominación, como los nuevos estatutos de la entidad (Decreto 204/2013). Las transformaciones impulsadas en la ley han cumplido su objetivo, ya que desde Septiembre de 2014, tras superar la evaluación pertinente, Unibasq es miembro 
de pleno derecho de ENQA (the European Association for Quality Assurance in Higher Education), y desde finales de 2014 está registrada en EQAR (European Quality Assurance Register for Higher Education).

Con la nueva configuración legal, Unibasq es un ente público de derech o privado adscrito al departamento del Gobierno Vasco competente en materia de universidades, con personalidad jurídica propia y plena capacidad de obrar. Esto supone que siendo una entidad pública, mantiene su independencia o personalidad jurídica propia y diferenciada de la Administración Autonómica de la que deriva, y que somete su actividad en las relaciones externas, con carácter general, a las normas del Derecho civil, mercantil y laboral que le sean aplicables, excepto en los actos de evaluación, acreditación o certificación y los que impliquen el ejercicio de potestades públicas, los cuales quedan sometidos al Derecho público. De este modo, en todas las evaluaciones quedan garantizados todos los derechos de las personas e instituciones implicadas.

El objetivo final de Unibasq es la mejora de la calidad y la competitividad del Sistema Universitario Vasco (en adelante, SUV), considerando el marco español e internacional. Para ello, se le encomienda la evaluación y certificación institucional (de los sistemas de gestión de las universidades y sus centros), la de las titulaciones que ofrecen (autorización-verificación, seguimiento y renovación de la acreditación), así como la evaluación y acreditación de su personal docente e investigador, tanto para el acceso a figuras contractuales que permitan su promoción profesional, como a efectos de concesión de complementos retributivos asociados a méritos investigadores, docentes y de gestión. Asimismo, se potencia su carácter asesor, atribuyéndole la función de "proporcionar información y criterios a las administraciones públicas, a las universidades y a otros agentes educativos o científico-tecnológicos en sus procesos de toma de decisiones relativas a las funciones que tiene encomendadas la agencia", y se le obliga a informar a la sociedad sobre el sistema universitario vasco y sobre las propias actividades de la agencia. Por otra parte, como se ha mencionado anteriormente, aunque las actividades anteriores se desarrollan principalmente en el ámbito del SUV, Unibasq también puede realizar evaluaciones, acreditaciones y certificaciones fuera de la Comunidad Autónoma de Euskadi, mediante acuerdos previamente suscritos con entidades extranjeras.

\subsection{Estructura organizativa}

Para garantizar la independencia y objetivida d de las evaluaciones y de las actividades de Unibasq, su estructura orgánica es bicéfala, separando el órgano de gobierno y gestión de la Agencia (Consejo de Gobierno), del órgano técnico que decide los protocolos o criterios a tener en consideración en cada una de las evaluaciones (Comisión Asesora).

El Consejo de Gobierno y la Directora o Director constituyen los órganos de gobierno de la agencia. En lo que se refiere al primero, está presidido por la consejera o el consejero del Gobierno Vasco competente en materia de Universidades, la viceconsejera o el viceconsejero competente en materia de universidades, la directora o director de la agencia, los tres Rectores o Rectoras de las universidades vascas, otra persona con 
prestigio académico reconocido nombrada por el Rector de la Universidad del País Vasco, una o un estudiante, y seis personas nombradas por el Consejo Vasco de Universidades (dos de ellas deben desarrollar su labor fuera del ámbito universitario; y otras tres deben trabajar fuera de la Comunidad Autónoma de Euskadi, -y más específicamente, una de esas tres, debe trabajar fuera del territorio español, y las otras dos, fuera de la comunidad universitaria-). El Consejo de Gobierno es responsable de la gobernanza de la agencia en términos estratégicos y estructurales, por ejemplo aprobando el anteproyecto de presupuestos de la agencia, ratificando su contabilidad, aprobando los plantes estratégicos y anuales de gestión, nombrando a la directora o director, etc. Sus miembros no podrán en ningún caso formar parte de ningún órgano técnico de evaluación ni acreditación, ni formar parte de ningún panel de expertos y expertas que vayan a realizar actividades evaluadoras. Es más, la composición garantiza que incluso para las funciones de gobierno y gestión se tengan en cuenta tanto las opiniones de las instituciones participantes en el SUV (Gobierno Vasco y Universidades), como de personas expertas ajenas al mismo. De hecho, existe un equilibro entre ambos tipos de perfil entre los miembros del Consejo.

Por su parte, la directora o director es nombrada por el Consejo de Gobierno a propuesta de su presidente o presidenta atendiendo a criterios de competencia y experiencia por un período de cuatro años renovables por otros cuatro si el Consejo le otorga de nuevo la confianza. Le corresponde en general cualquier función relacionada con la dirección, gestión ordinaria y representación de la agencia. En particular, propone al Consejo la persona que va a ocupar el cargo de adjunto a la dirección, y nombra y cesa a las personas integrantes de los órganos técnicos (Comisión Asesora y Comités de evaluación).

En cuanto a los órganos técnicos de evaluación y certificación, la Comisión Asesora es el superior. Está compuesta por la directora o director de la Agencia, que la preside, y diez personas de reconocido prestigio en el ámbito universitario o de evaluación, nombradas por la directora o director de la agencia. La mayoría de los miembros de esta comisión tienen que ser ajenos al sistema universitario vasco, debiendo al menos tres de sus miembros desarrollar su labor fuera de España. Además, incluye a una o un estudiante. Con ello se pretende que en sus decisiones prevalezcan argumentos científico- técnicos reconocidos y compartidos a nivel nacional e internacional en la evaluación de profesorado, de titulaciones y de las instituciones, lo que garantiza la credibilidad de las decisiones evaluadoras y certificadoras de Unibasq. Aprueba los Protocolos que fijan los criterios de evaluación y el peso que debe darse a cada uno de ellos en las decisiones. Por otra parte, es también la Comisión Asesora la que presenta propuestas a la directora o director para nombrar a los miembros de los Comités de Evaluación, que son quienes finalmente realizarán las evaluaciones o certificaciones desarrollando y aplicando los protocolos anteriormente mencionados.

Cada Comité de Evaluación está compuesto por:

- Académicos de reconocido prestigio. Como norma fundamental que garantiza la imparcialidad y la lejanía en la toma de decisiones, en cada Comité serán mayoría los académicos ajenos a las universidades del SUV.

- Estudiantes de las áreas a evaluar, en la evaluación de los programas y activi- 
dades que tengan un impacto directo sobre el alumnado. Dado que el alumnado es el principal usuario del sistema universitario, son esenciales sus opiniones sobre el mismo. Hasta el momento se ha entendido que tienen "un impacto directo sobre el alumnado" los programas de evaluación de titulaciones y de evaluación institucional, puesto que son usuarios directos de estos servicios. En cambio, quedan al margen las evaluaciones individuales de profesorado a fin de conseguir su acreditación o complementos retributivos por actividades investigadoras, docentes y de gestión. Aunque la calidad del profesorado repercute en las titulaciones y en los servicios prestados por un centro universitario, el impacto sobre el alumnado es indirecto. Estos representantes del alumnado son designados por el órgano específico de participación de los estudiantes universitarios de Euskadi.

- Profesionales de reconocido prestigio de los ámbitos de conocimiento que se deben evaluar en los programas de evaluación de las titulaciones. Con ello se pretende que agentes del entorno socioeconómico en el que están destinados a desempeñar su actividad las personas egresadas de un título, participen en la evaluación de esa titulación opinando si su diseño y forma de implantación responde realmente a las necesidades de ese entorno.

Los distintos Comités de Evaluación existentes en Unibasq, así como las personas que los componen, están publicados en la página web de la Agencia (http//www.unibasq.org).

Toda la actividad desarrollada por Unibasq y por sus diferentes órganos se apoya en la actualidad en el trabajo de 12 personas ( 10 mujeres y 2 hombres). La directora, el director adjunto, la responsable de evaluación, la responsable de finanzas, cinco técnicos y técnicas de evaluación, dos auxiliares administrativas y una secretaria de dirección.

\section{Evolución de la actividad realizada por la agencia en el periodo 2004-2015}

\subsection{El sistema universitario vasco}

Para entender completamente la forma de funcionamiento de Unibasq es necesario presentar el contexto principal de su actuación en este periodo de actividad. EI SUV está compuesto de tres universidades, cada una de las cuales cuenta con varios campus y tienen su principal sede en territorio vasco. Además, cada una de las tres universidades vascas tiene un tipo diferente de titularidad:

- Universidad del País Vasco / Euskal Herriko Unibertsitatea - UPV/EHU es la única universidad pública del País Vasco y tiene tres campus situados en las tres provincias vascas: Gipuzkoa, Bizkaia y Araba.

- La Universidad de Deusto (Deustuko Unibertsitatea) es una universidad privada sin ánimo de lucro, perteneciente a la Sociedad de Jesús. Cuenta con dos campus en el País Vasco, uno en Bilbao y otro en Donostia-San Sebastián, además de una sede en Madrid (Deusto Business School).

- Mondragon Unibertsitatea es una universidad privada sin ánimo de lucro orga- 
nizada como entidad de propiedad cooperativa fundada en 1997 gracias a la fusión de diversos institutos educativos y formativos existentes previamente.

\begin{tabular}{|l|l|l|l|l|l|l|l|}
\hline Universidad & $\begin{array}{l}\text { Año de } \\
\text { fundaci- } \\
\text { ón }\end{array}$ & Tipo & $\begin{array}{l}\text { Gra- } \\
\text { dos }\end{array}$ & $\begin{array}{l}\text { Máste- } \\
\text { res }\end{array}$ & $\begin{array}{l}\text { Docto- } \\
\text { rado }\end{array}$ & $\begin{array}{l}\text { Profeso- } \\
\text { rado }\end{array}$ & $\begin{array}{l}\text { Estudian- } \\
\text { tes }\end{array}$ \\
\hline UPV/EHU & 1980 & Pública & 70 & 111 & 65 & 4450 & 42063 \\
\hline $\begin{array}{l}\text { Universidad } \\
\text { de Deusto }\end{array}$ & 1886 & $\begin{array}{l}\text { Privada, } \\
\text { sin ánimo } \\
\text { de lucro, } \\
\text { Iglesia }\end{array}$ & 26 & 52 & 8 & 572 & 8405 \\
\hline $\begin{array}{l}\text { Mondragon } \\
\text { Unibertsi- } \\
\text { tatea }\end{array}$ & 1997 & $\begin{array}{l}\text { Privada, } \\
\text { sin ánimo } \\
\text { de lucro, } \\
\text { cooperati- } \\
\text { va }\end{array}$ & 15 & 15 & 3 & 345 & 4102 \\
\hline
\end{tabular}

Tabla 1. Composición del Sistema Universitario Vasco (Datos SIIU (Sistema Integrado de Información Universitaria)-Curso 2014-2015)

Esta diversidad de titularidades y de filosofías subyacentes otorga al SUV una personalidad única, basada en una mezcla de cooperación y competencia entre 3 universidades firmemente comprometidas con el papel que desempeñan en la sociedad y economía vascas. Su distinta estructura, finalidad y filosofía hace que sus características sean notoriamente diversas y complementarias. De hecho, en el Anuario Regional de Eurostat del año 2014, los datos referentes a "personas de entre 25-64 años que han completado estudios universitarios" muestran de manera clara que el País Vasco es una de las regiones europeas con mayores promedios, situándose tras Londres Central entre las regiones con el mayor porcentaje de titulados universitarios entre 30 y 34 años $(61,7 \text { frente a } 73,1 \%)^{1}$.

\subsection{Estudios y titulaciones universitarias.}

Desde que en el año 2007 el sistema universitario español tuvo que llevar a cabo la adecuación a las características comunes del Espacio Europeo de Educación Superior (EEES), la estructura de los estudios universitarios en España ha estado inmersa en un continuo proceso de transformación.

En el sistema anterior, el título de mayor nivel era la licenciatura, un primer grado largo con una duración oficial de 4 o 5 años; en algunos campos también existía una titulación superior con una duración algo inferior, en concreto, de 3 años, que otorgaba una diplomatura o ingeniería técnica en el campo correspondiente; y no existían másteres oficiales en España. Los programas de doctorado consistían, principalmente, en

1 http://ec.europa.eu/eurostat/documents/3217494/5785629/KS-HA-14-001-EN.PDF/e3ae3b5c-b104-47e9-ab80-36447537ea64 
unos cursos de doctorado y la preparación y defensa de una tesis. En el nuevo sistema, implantado para adaptarse al EEES, las universidades españolas ofrecen ahora tres ciclos similares a otros países: Grados, Másteres y Doctorados.

\section{ECTS}

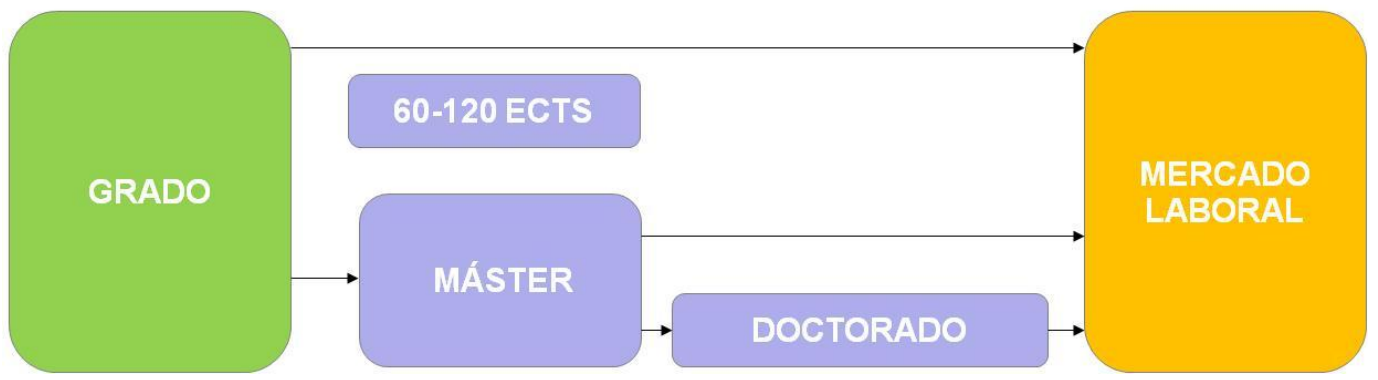

Figura 1. Organización de las titulaciones oficiales en España

En el curso académico 2014-2015 se matricularon casi 55.000 estudiantes en las universidades vascas entre estudiantes de grado y primer y segundo ciclo, y estudiantes de máster; el $77 \%$ del total del alumnado universitario se matriculó en la universidad pública, es decir, en la UPV/EHU.

En la siguiente figura puede observarse la evolución en la matriculación de estudiantes en las tres universidades del SUV que se ha mantenido en los últimos cursos académicos e incluso ha ido aumentando en el caso de Mondragon Unibertsitatea que muestra una evolución positiva en el número de matriculaciones. Téngase presente que es una universidad más joven y aún en proceso de expansión.

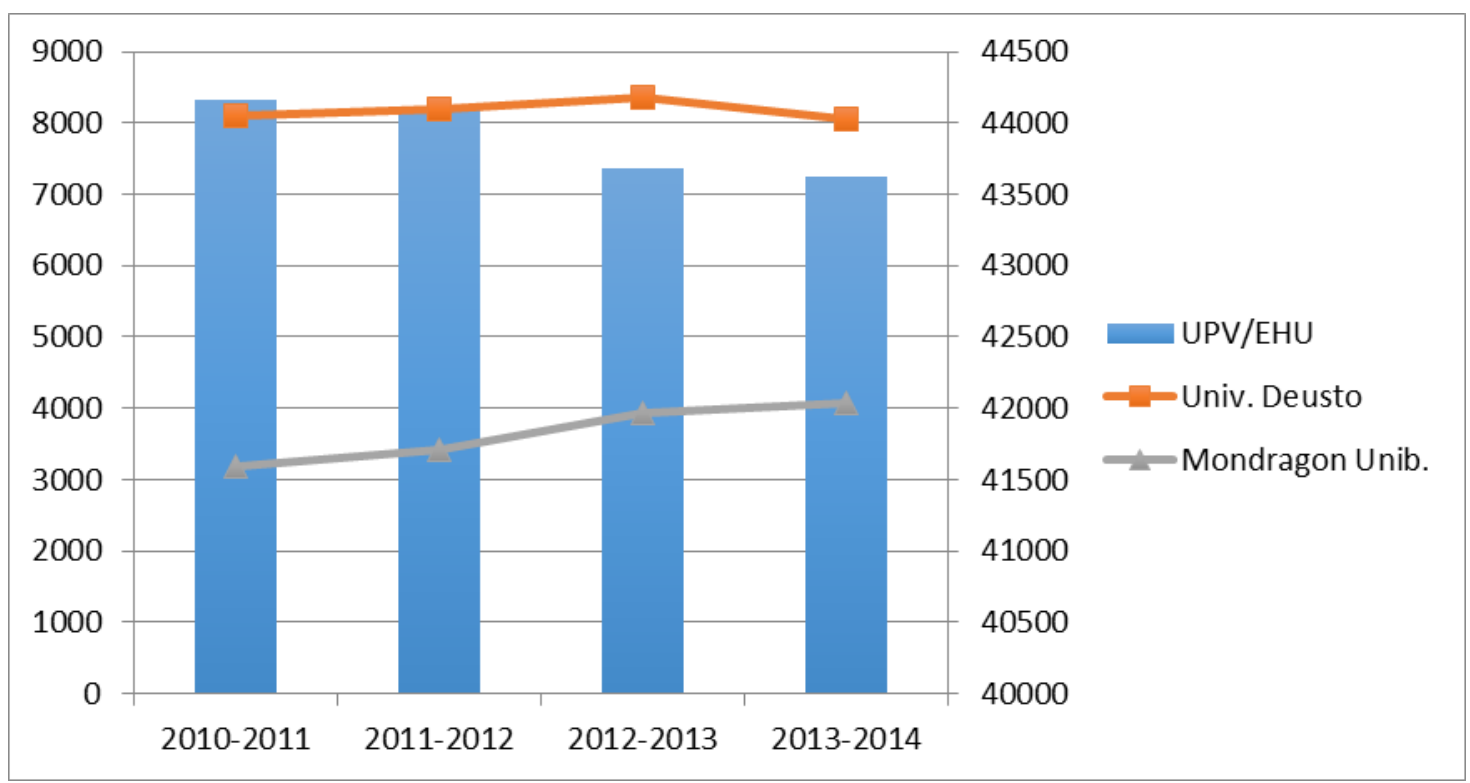

Figura 2. Evolución del número de estudiantes matriculados en las tres universidades del SUV hasta el curso 2013/2014 (Los datos de la UPV/EHU corresponden al eje de la derecha). Fuente: SIIU 


\subsection{Actividad de la Agencia.}

Según sus estatutos (Decreto 204/2013), Unibasq se ocupa de las siguientes actividades:

a) La evaluación de las enseñanzas universitarias.

b) La evaluación institucional y su certificación.

c) La acreditación de las enseñanzas en el marco europeo de la calidad.

d) La evaluación y acreditación del personal docente e investigador.

e) La evaluación de los méritos individuales de investigación del personal docente e investigador para la asignación de complementos retributivos y para la acreditación de acuerdo con las competencias atribuidas legalmente, así como la evaluación del cumplimiento de los objetivos que establezca el Gobierno Vasco para la actividad investigadora.

f) La evaluación de las actividades de la investigación.

g) La elaboración de estudios orientados a la mejora y a la innovación de modelos de evaluación, certificación y acreditación.

h) La promoción de la evaluación y de la comparación de criterios de calidad en el marco europeo e internacional.

i) El asesoramiento sobre cuestiones relativas a la calidad del sistema universitario vasco en el ámbito de sus funciones.

j) Cualquier otra función en relación con su objeto y su ámbito de actuación que se le encomiende por parte del Departamento del Gobierno Vasco competente en materia de universidades.

Las actividades de evaluación iniciadas por Unibasq hasta la fecha se pueden dividir en dos tipos principales: actividades desarrolladas por la propia Agencia, y actividades desarrolladas en colaboración con otras agencias españolas para la garantía de la calidad.

\subsubsection{Actividades desarrolladas por la propia Agencia}

\section{Evaluación de titulaciones e instituciones:}

- Evaluación previa de todos los nuevos programas de estudios de las universidades vascas desarrollados de acuerdo con la estructura de titulaciones del EEES; antes de que el Gobierno Vasco autorice la impartición de un nuevo título oficial, es obligatorio contar con la evaluación positiva por parte de Unibasq. Además de ese requisito autonómico, existe otro requisito a nivel nacional, se- 
gún el cual es obligatorio obtener una evaluación positiva previa (denominada verificación) para que cualquier programa nuevo se convierta en oficial, es decir, tenga validez en todo el territorio español y se inscriba en el Registro de Universidades, Centros y Títulos (RUCT). Unibasq es competente para realizar la verificación de nuevas titulaciones a partir de su entrada en ENQA y su registro en EQAR a finales de 2014.

Desde 2006 se han evaluado más de 300 programas para la emisión del informe previo a su autorización, de los que aproximadamente un $95 \%$ ha obtenido una evaluación favorable.

\begin{tabular}{|l|l|l|}
\hline \multicolumn{2}{|l|}{ TÍTULOS EVALUADOS } & POSITIVOS \\
\hline $\mathbf{2 0 0 6}$ & 39 & 38 \\
\hline $\mathbf{2 0 0 7}$ & 24 & 12 \\
\hline $\mathbf{2 0 0 8}$ & 34 & 33 \\
\hline $\mathbf{2 0 0 9}$ & 92 & 90 \\
\hline $\mathbf{2 0 1 0}$ & 29 & 28 \\
\hline $\mathbf{2 0 1 1}$ & 16 & 15 \\
\hline $\mathbf{2 0 1 2}$ & 25 & 24 \\
\hline $\mathbf{2 0 1 3}$ & 85 & 84 \\
\hline $\mathbf{2 0 1 4}$ & 18 & 18 \\
\hline
\end{tabular}

Tabla 2. Títulos evaluados para la emisión de informe previo a su autorización.

En 2015 se ha comenzado con la evaluación para la verificación de titulaciones oficiales y se han emitido informes finales de verificación de 3 titulaciones de máster.

- Seguimiento de la implantación de los títulos oficiales del Sistema Universitario Vasco. Una vez que los títulos oficiales inscritos en el RUCT se han puesto en marcha, Unibasq supervisa que los mismos cumplen el proyecto establecido en el plan de estudios verificado. Desde 2010 que se desarrolló la convocatoria piloto se han emitido 143 informes de seguimiento;

- Renovación de la acreditación de los títulos oficiales; se trata de una evaluación periódica de cómo se desarrolla la implantación de los títulos oficiales incluyendo una visita de expertos externos a la universidad. Este programa se puso en marcha en 2014 con una convocatoria piloto en la que participaron 8 titulaciones. Desde entonces se han evaluado las titulaciones que se recogen en la siguiente tabla, los informes definitivos pueden consultarse en la página web de la agencia:

\begin{tabular}{|l|l|l|l|}
\hline Convocatoria & UPV/EHU & Mondragon Unib. & Univ. Deusto \\
\hline $\mathbf{2 0 1 4}$ (octubre) & 51 másteres & 2 másteres & 13 másteres \\
\hline $\mathbf{2 0 1 5}$ (mayo) & 63 grados & -- & -- \\
\hline $\mathbf{2 0 1 5}$ (octubre) & 10 másteres & $\begin{array}{l}4 \text { grados } \\
6 \text { másteres }\end{array}$ & 6 másteres \\
\hline
\end{tabular}

Tabla 3. Títulos evaluados en las convocatorias para la renovación de la acreditación de títulos oficiales. 
- Evaluación externa de las propuestas de nuevos títulos propios de la UPV/EHU. Desde 2007 se han evaluado 85 propuestas de las cuales 71 han obtenido un informe favorable;

- Informes sobre el cumplimiento de los acuerdos (contratos-programa) suscritos por el departamento del Gobierno Vasco competente en materia de universidades y cada una de las universidades del Sistema Universitario Vasco, en relación con los objetivos establecidos por el Gobierno en el Plan Universitario del País Vasco. En el último periodo evaluado se recogían los contratos-programa referidos a:

- Formación reglada

- Formación no reglada

- Investigación

- Transferencia

- Equidad, eficacia y proyección social

La evaluación consiste en revisar las acciones realizadas en cada contrato-programa y los indicadores correspondientes frente a los objetivos y metas establecidas.

Evaluación del personal académico en función de su labor docente e investigadora:

- Acreditación del Personal Docente e Investigador (PDI), para que puedan optar a puestos de trabajo con contrato laboral en universidades públicas, así como a puestos de profesorado doctor en universidades privadas del Sistema Universitario Vasco. Desde 2008 se han evaluado, en diferentes convocatorias, las solicitudes que pueden observarse en la siguiente tabla:

\begin{tabular}{|l|l|l|}
\hline CONVOCATORIAS & $\begin{array}{l}\text { Solicitudes } \\
\text { evaluadas }\end{array}$ & Favorables \\
\hline 2008 & 972 & 630 \\
\hline 2009 & 1278 & 836 \\
\hline 2010 & 1264 & 924 \\
\hline 2011 & 881 & 363 \\
\hline 2012 & 386 & 154 \\
\hline 2013 & 526 & 199 \\
\hline 2014 & 510 & 219 \\
\hline 2015 & 273 & 131 \\
\hline TOTAL & 6090 & 3456 \\
\hline
\end{tabular}

Tabla 4. Solitudes evaluadas y resultados favorables en la acreditación del PDI.

- Evaluación del Personal Docente e Investigador de la UPV/EHU para asignar los 
complementos retributivos establecidos para ese fin por el Gobierno de la Comunidad Autónoma Vasca. Se evalúa la actividad docente, investigadora y de gestión. Hasta 2011 se habían evaluado 3983 solicitudes. En 2015 se ha publicado una nueva convocatoria en la que se han recibido 1883 solicitudes.

- Evaluación del personal docente e investigador laboral permanente de la UPV/EHU para el reconocimiento de los "sexenios", es decir, para la validación de periodos de seis años de actividad investigadora. Desde 2010 se han evaluado 781 solicitudes, obteniendo un resultado favorable el $82 \%$ de ellas.

\subsubsection{Actividades en cooperación con otras agencias}

Evaluación Institucional

- DOCENTIA - Programa de apoyo a las universidades para el diseño de procedimientos propios para la evaluación de la actividad docente de su profesorado. DOCENTIA se diseñó conjuntamente con la agencia nacional (ANECA) y todas las agencias regionales de garantía de la calidad existentes en España. Las tres universidades del SUV han participado en este programa, con diferentes niveles de implantación.

- AUDIT - Programa de evaluación y certificación de los sistemas de garantía interna de calidad de los centros universitarios del Sistema Universitario Vasco. AUDIT se diseñó conjuntamente entre ANECA y las agencias de acreditación de Cataluña (AQU Catalunya) y Galicia (ACSUG); Unibasq se unió al programa en 2007. Se han evaluado los diseños de los sistemas de calidad de 41 centros de las 3 universidades y desde 2013 se está evaluando la implantación real de estos sistemas. En estos momentos, 9 centros de las universidades del País Vasco disponen del certificado de la implantación de su Sistema de Garantía Interna de Calidad.

\section{Nuevos retos: Plan Estratégico 2016-2019}

\subsection{Misión, visión y valores}

MISIÓN

Unibasq-Agencia de Calidad del Sistema Universitario Vasco-, es un ente público de derecho privado cuyo objeto es contribuir a la mejora e innovación del sistema universitario vasco, satisfaciendo estándares internacionales de calidad y atendiendo al interés que la sociedad tiene por la educación superior. Para ello asesora, evalúa y acredita tanto las propias instituciones que lo componen, como sus titulaciones y profesorado, de forma independiente y objetiva. Su actividad está reconocida por el cumplimiento de los criterios y directrices para la calidad del EEES, siendo miembro de pleno derecho de ENQA y estando registrada en EQAR, lo que permite el desarrollo de sus actividades en cualquier sistema universitario que así lo establezca y el reconocimiento de sus ac- 
tividades por el resto de sistemas europeos.

VISIÓN

Unibasq quiere ser reconocida por las instituciones de educación superior, la comunidad universitaria y la sociedad como un agente que ayuda a mejorar la calidad del sistema universitario vasco, así como a su internacionalización y proyección social.

Pretende ser un interlocutor entre las universidades vascas, los grupos de interés o usuarios del sistema, las instituciones que deciden la política universitaria y los agentes externos que se nutren de los resultados universitarios.

Asimismo quiere ser reconocida a nivel internacional como agencia asesora y aseguradora de la calidad universitaria, principalmente en los países de Europa y Latinoamérica de mayor interés para las universidades vascas, con el fin de apoyar su internacionalización.

VALORES

1. Independencia en el cumplimiento de sus tareas.

2. Responsabilidad, profesionalidad y ética de las personas.

3. Eficacia y eficiencia mediante una gestión innovadora, que optimice los recursos disponibles.

4. Objetividad e integridad en el desarrollo de los procesos de evaluación.

5. Transparencia y rendición de cuentas ante la sociedad.

6. Vocación de servicio y de mejora continua.

7. Respeto a la autonomía y especificidad de las universidades.

8. Fomento de la cooperación con las instituciones de educación superior, la administración, y otras agencias y organismos nacionales e internacionales con intereses comunes.

\subsection{Ejes estratégicos y principales objetivos}

El Plan Estratégico de Unibasq (2016-2019) recoge 4 ejes estratégicos en los que la agencia centrará su actividad para poder desarrollar su misión en los próximos cuatro años:

1. Eje 1: Servicios: Asesoramiento y Evaluación

2. Eje 2: Proyección Social: Comunicación, Colaboración con Instituciones, Otras Agencias de Calidad y con Agentes Sociales

3. Eje 3: Internacionalización de la Agencia en Apoyo al Sistema Universitario Vasco

4. Eje 4: Organización Interna: Dirección, Planificación, Recursos Humanos

Tal y como se menciona en la introducción, Unibasq realiza el Plan Estratégico teniendo en cuenta un diagnóstico de la situación, no sólo desde la propia evolución, sino también a partir de las reflexiones realizadas desde otras experiencias y otros sistemas universitarios. 
Esquemáticamente, los principales objetivos del plan son:

Eje 1: Simplificación de protocolos de evaluación e identificación de un número reducido pero suficiente de indicadores que permitan medir adecuadamente la actividad académica, con especial atención a la calidad docente del profesorado y a los resultados del aprendizaje.

Eje 2: Mayor proyección social de las medidas de calidad universitariay, en consecuencia, mejora de la visibilidad de las buenas prácticas, mediante la comunicación y la promoción de reconocimientos de sellos de calidad con repercusión social. Refuerzo de la participación de estudiantes y grupos de interés en los procesos de evaluación y asesoramiento. Este refuerzo conduce a mayor corresponsabilidad y mayor identificación con la labor realizada en las universidades.

Eje 3: Colaboración de Unibasq con agencias de otros países que sean de interés para las universidades vascas para favorecer la movilidad y la colaboración entre las universidades.

Eje 4: Mejora de los procesos internos de la Agencia y de la proyección social de la actividad realizada.

\section{Bibliografía}

Calderón, C. y Escalera, G. (2008) La evaluación de la docencia ante el reto del Espacio Europeo De Educación Superior (EEES), Educación XX1. 11, 237-256.

Cartwright, M. J. (2007) The rhetoric and reality of "quality" in higher education. An investigation into staff perceptions of quality in post-1992 universities, Quality Assurance in Education 15:3, 287-301.

Casanova, M.A. (2015) La Supervisión, Eje del Cambio en los Sistemas Educativos, REICE. Revista Iberoamericana sobre Calidad, Eficacia y Cambio en Educación, 2015, 13(4), 7-20.

$\begin{array}{lllll}\text { EUROSTAT (2014) } & \text { Eurostat regional }\end{array}$ http://ec.europa.eu/eurostat/documents/3217494/5785629/KS-HA-14-001EN.PDF/e3ae3b5c-b104-47e9-ab80-36447537ea64)

Ion, G. y Cano, E. (2011) Assessment practices at Spanish universities: from a learning to a competencies approach, Evaluation \& Research in Education, 24:3, 167-18.

Jackson, N. J. (2000) The concept of programme specification and its application in the new quality assurance framework, Quality Assurance in Education 8:4, 164-172.

Lomas, L. (1999), The culture and quality of higher education institutions: examining the links, Quality Assurance in Education 7:1, 30-34. 
Molero, D. (2004). La evaluación de la docencia en la Universidad. Jaén: Universidad de Jaén, Servicio de Publicaciones.

Rue, J. (2009) El cambio en la Universidad, sus epistemologías y consecuencias de las mismas, Revista Complutense de Educación, Vol. 20 Núm. 2 (2009) 295-317.

Ruiz, J. (2005). La evaluación de la docencia en los planes de mejora de la universidad. Educación XX1, 8, 87- 102 